\title{
Long term in vivo stability and frequency response of polyimide based flexible array probes
}

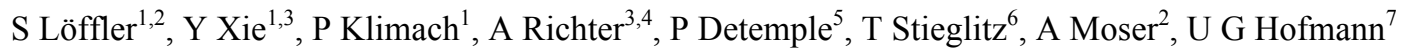 \\ ${ }^{1}$ Institute for Signal Processing, University of Lübeck, 23538 Lübeck, Germany \\ ${ }^{2}$ Department of Neurology, University of Lübeck, 23538 Lübeck, Germany \\ ${ }^{3}$ Graduate School for Computing in Medicine and Life Sciences, University of Lübeck, 23538 Lübeck, Germany \\ ${ }^{4}$ Group of Cell Differentiation, Fraunhofer-Institution for Marine Biotechnology, 23538 Lübeck, Germany \\ ${ }^{5}$ Institut für Mikrotechnik Mainz, 55129 Mainz, Germany \\ ${ }^{6}$ Institute for Microsystem Technology, Albert-Ludwigs-University of Freiburg, 79106 Freiburg, Germany \\ ${ }^{7}$ Neuroelectronic Systems, Department of Neurosurgery, University Medical Center Freiburg, 79106 Freiburg, Germany
}

Structure: 1. Introduction / 2. Methods / 3. Results / 4. Conclusion / 5. References

\begin{abstract}
The frequency response of chronically implanted neuronal probes determines their usability in neural interface applications. Frequency range and noise content of the measured signal depend on the impedance of the electrode-electrolyte interface. After implantation into cerebral tissue, the impedance at the electrode-tissue interface changes. We used flexible, polyimide based multisite microprobes and measured the impedance at the probe contact sites in ionic electrolyte and brain tissue over a long time range. Adapted equivalent circuit models were used to characterize the components of the electrode-electrolyte interface and the electrode-tissue interface after implantation. The RC elements in the equivalent circuit defining the transition between electrolyte and brain tissue in the acute and chronic stage were identified. After implantation, an additional RC element was introduced. Even after implantation, characteristic $1 \mathrm{kHz}$ impedances were lower than $500 \mathrm{k} \Omega$.
\end{abstract}

\section{Introduction}

Current development in neuroprosthetics is focused towards small sized MEMS based microelectrodes, which facilitate extracellular recordings in neuronal tissue via multiple contact sites [1]. Extracellular potentials in neuronal tissue represent the spatial summation of local electric fields resulting from transmembrane currents at the neuronal membrane [2]. Originating from high frequency neuronal discharge events during action potential generation and low frequency events during synaptic integration, extracellular potentials cover a wide frequency range [3]. Thus, to interpret measurements, it is crucial to determine the frequency response of neuronal recording electrodes. Depending on the properties of the electrode-electrolyte or electrode-tissue interface, neural probes display a frequency response resulting in a shift in amplitude and phase which is dependent on the frequency of the measured signal [4]. The frequency response of an electrode in the respective electrolyte is described by the complex impedance $Z$. Equivalent circuit models have been used to describe the impedance $Z$ at the electrode-electrolyte interface [5]. Based on the assumption that an ionic double layer (HeLMHOLZ double layer) forms at the surface of a polarized electrode in an electrolyte solution, a simple RANDLES cell equivalent circuit can be used to characterize the impedance of an electrode-electrolyte interface [6]. The RANDLES cell equivalent circuit consist of a double layer capacitance $\mathrm{C}_{\mathrm{dl}}$ in parallel with a charge transfer resistance $\mathrm{R}_{\mathrm{ct}}$ and a serial resistance $R_{s}$. When the electrochemical reactions at the interface are not limited by diffusion, the WARBURG impedance accounting for mass transfer limitations, can be omitted in the model [7].

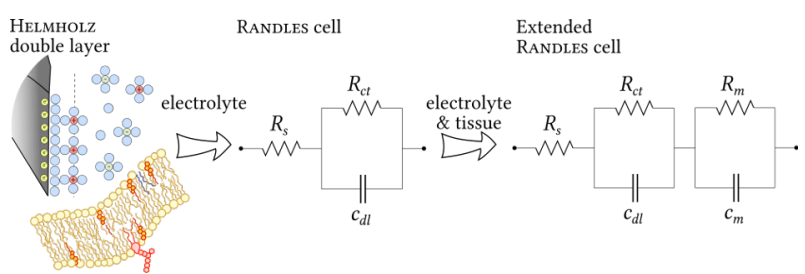

Image 1 Equivalent circuit models used in the current study to describe electrode-electrolyte and electrode-tissue interface. The RANDLES cell model is used to describe the electrode-electrolyte interface. It implies a serial spreading resistance $R_{s}$ as well as a parallel RC element with charge transfer resistance $R_{s}$ and double layer capacitance $C_{d l}$. To characterize the electrode-tissue interface, the RANDLES cell model is extended by another parallel RC element with the membrane resistance $R_{m}$ and the membrane capacitance $\mathrm{C}_{\mathrm{m}}$.

When an electrode is implanted into cerebral tissue, the impedance characteristics will change requiring an adapted equivalent circuit model to describe the frequency re- 
sponse of the electrode-tissue interface [8]. Further, it is reported that the composition of the tissue around the electrode changes after implantation [9]. Thus, the equivalent circuit model for the electrode-tissue interface after implantation has to be expanded. Yousif et al. used a parallel $\mathrm{RC}$ element representing the peri-electrode space and another parallel RC element representing the neuronal tissue [8]. In the chronic stage, after one week of implantation, a so-called layer of giant cells has been reported to surround the electrode [9].

In the current study, we measured the frequency response of three different types of microprobes with comparable sizes in saline electrolyte. We compared the impedance of flexible, polyimide based, batch produced probes with the impedance of rigid, metal based, precision mechanically fabricated probes. Here, the RANDLES cell was used to describe the electrode-electrolyte interface (Image 1) . Further, we recorded impedance spectra at the contact sites of flexible, polyimide based probes after implantation in the rat brain for up to 180 days. Here, we used an extended RANDLES cell model with an additional parallel RC element consisting of membrane resistance $R_{m}$ and membrane capacitance $C_{m}$ to describe the characteristics of the electrode-tissue interface (Image 1).

\section{Methods}

Three different types of microprobes were used in the current study (Image 2). Rigid, metal based probes were produced by precision mechanics from a hollow stainless steel shaft and insulated gold microwires. A lateral array of contact sites was formed by the ends of the gold leads, embedded into the shaft. The rigid probes featured seven recording sites with $3850 \mu \mathrm{m}^{2}$ in a lateral array along the probe shaft over a distance of $750 \mu \mathrm{m}$ with a centre-tocentre distance of $125 \mu \mathrm{m}$, starting $400 \mu \mathrm{m}$ from the tip. A $11300 \mu \mathrm{m}^{2}$ stimulation site was located at the probe tip (Image 2 (A)). Flexible array probes for impedance measurement in saline electrolyte were microfabricated from electro-spun poly-imide. Three stimulation sites with 3600 $\mu \mathrm{m}^{2}$ in the middle of the shaft and six off-centered recording sites with $400 \mu \mathrm{m}^{2}$ were produced by gold vapor deposition and subsequent galvanical reinforcement.

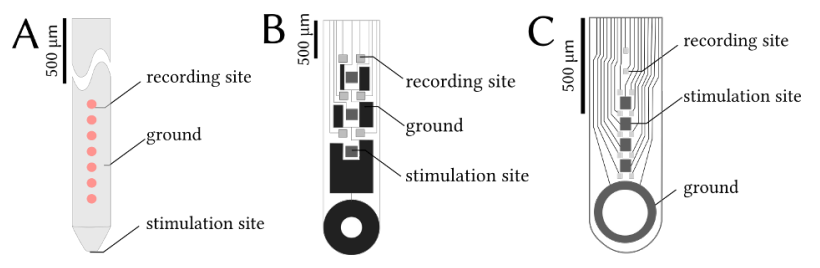

Image 2 Probes for in vitro measurements in saline electrolyte. Metal based rigid probe with 7 recording sites and one stimulation site at the tip, the probe shaft serving as ground (A). Polyimide based, flexible probe with 6 recording sites and 3 stimulation sites. Large gold patches serve as ground (B). Polyimide based, flexible probe with 12 recording sites and 4 stimulation sites. A gold ring at the tip serves as ground (C).
The probes featured alternating recording and stimulation sites as well as large surface ground contacts and a punch hole at the very tip to facilitate tissue insertion (Image 2 (B)). Another type of flexible, polyimide based probe was used for impedance measurements in saline electrolyte and was implanted in freely moving animals in vivo. The probes featured 12 recording sites with $225 \mu \mathrm{m}^{2}$ aligned in two columns with $105 \mu \mathrm{m}$ longitudinal and $60 \mu \mathrm{m}$ transversal spacing along a $405 \mu \mathrm{m}$ diameter shaft. Four 2500 $\mu \mathrm{m}^{2}$ stimulation sites were placed between the two recordings columns (Image 2 (C)).

The impedance $Z$ and phase angle $\Phi$ were measured at all probes sites using an LCR-800 impedance spectrometer (Good Will Instruments Co., Ltd). Measurements were performed using a three electrode setup with a large area counter electrode and a non-polarizable reference electrode whereas the respective probe contact site was used as working electrode.

In the in vitro setting, a platinum counter electrode and an $\mathrm{Ag} / \mathrm{AgCl}$ reference electrode were immersed in a beaker filled with $0.9 \%$ saline together with the contact site under test. The solution was constantly agitated using a magnetic stirrer. In the in vivo setting, the ground contact sites were used as counter electrodes and the reference electrode was represented by a skull screw in the rat's cranium. Impedance spectra were obtained by measuring amplitude and phase shift of a sinusiodal signal with $0.01 \mathrm{~V}$ amplitude in a frequency range between 0.1 and $100 \mathrm{kHz}$. Mean values and standard deviations were calculated. The data were fitted to the respective equivalent circuit model. All procedures with animals were reviewed and approved by the University of Lübeck and the Ministry for Agriculture, the Environment and Rural Areas, Schleswig-Holstein, Germany and were conducted in accordance with the NIH guide for the Care and Use of laboratory animals. Male Wistar rats were used and housed separately under standard lightning conditions ( $12 \mathrm{~h}$ light-dark cycle, lights on at $06: 00 \mathrm{am}), 22{ }^{\circ} \mathrm{C}$ and $40 \%$ humidity with free access to food and water. A stereo-taxic surgery was carried out for precise probe insertion. Anaesthesia was given in form of inital isoflurane inhalation and subsequent i.p. injection with $80 \mathrm{mg} / \mathrm{kg}$ ketamine (Ketavet ${ }$, Pfizer) and $1 \mathrm{mg} / \mathrm{kg}$ xylazine (Rom-pune ${ }^{\circledR}$, Bayer). The probes were implanted into the tha-lamic ventral posteromedial nucleus at $\mathrm{AP}+0.56, \mathrm{ML}-0.28, \mathrm{DV}-0.6$ (in $\mathrm{cm}$ ) relative to the interaural point. A glass fiber with $250 \mu \mathrm{m}$ diameter was used as support during insertion and was removed after implantation. The impedance was measured in awake and freely behaving animals, six months after probe implantation.

\section{$3 \quad$ Results}

The frequency response at the recording- and stimulation sites of the rigid, metal based and flexible, polyimide based probes in saline electrolyte was determined and is shown as Bode- and Nyquist plot (Image $3 \mathrm{a}, \mathrm{b}$ ).

The data were fitted using the RANDLES cell equivalent circuit ommiting the effect of diffusion (Iamge 1). The values 
a) rigid probe in electrolyte
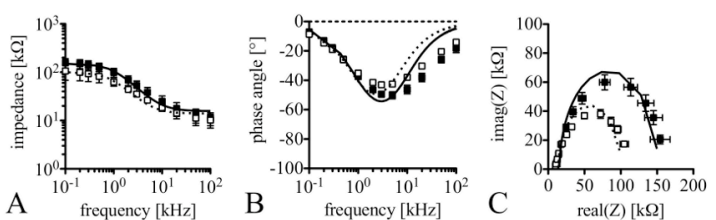

b) flexible probe in electrolyte
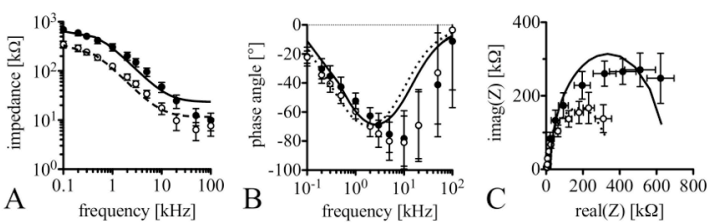

c) flexible probe in tissue
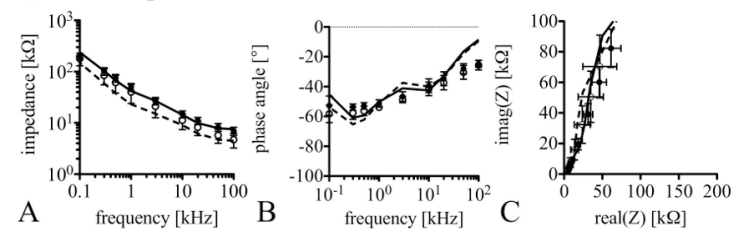

Image 3 caption over 2 lines (10/12 pt) Impedance spectra shown in BODE- $(\mathrm{A}+\mathrm{B})$ and NYQUIST $(\mathrm{C})$ representation. The data and the respective fits are shown for recording $(\bullet, \quad)$ and stimulation ( $(,--)$ sites. a) Rigid, metal based probe in saline electrolyte with RANDLES cell fit. b) Flexible, polyimide based probes in saline electrolyte with RANDLES cell fit. c) Flexible, polyimide based probes implanted tissue for 180 days, extended RANDLES cell fit.

of the RANDLES cell components were determined from the impedance spectra and RANDLES cell fits (Table 1). The spreading resistance $R_{S}$ was determined from the low fre quency asymptote in the BoDE plot and the lower intersection with the real axis in the NyQUIST plot.

Table 1 Values for the RANDLES cell components determined from the impedance spectra in BODE and NYQUIST representation.

\begin{tabular}{ccccc} 
& \multicolumn{2}{c}{ rigid probes } & \multicolumn{2}{c}{ flexible probes } \\
& recording & stimulation & recording & stimulation \\
\hline $\mathrm{R}_{\mathrm{s}}[\mathrm{k} \Omega]$ & $11.1 \pm 3.3$ & $10.2 \pm 3.2$ & $24.8 \pm 7.6$ & $7.7 \pm 2.9$ \\
$\mathrm{R}_{\mathrm{ct}}[\mathrm{k} \Omega]$ & $155.8 \pm 34.1$ & $106 . \pm 38.1$ & $692.3 \pm 73.2$ & $346.9 \pm 55.0$ \\
$\mathrm{C}_{\mathrm{dl}}[\mathrm{nF}]$ & $2.1 \pm 0.2$ & $2.2 \pm 0.1$ & $3.0 \pm 0.1$ & $5.1 \pm 1.3$
\end{tabular}

The charge-transfer resistance $\mathrm{R}_{\mathrm{ct}}$ was determined from the high frequency asymptote in the BoDE plot and the difference between higher and lower intersection with the real axis in the Nyquist plot. The double layer capacitance $\mathrm{C}_{\mathrm{dl}}$ was calculated from the NyQuisT Plot with $C_{d l}=1 /(2 \pi f R)$ whereas the real part of the impedance $\mathrm{Z}$ and the frequency at the apex were used.
Table 2 Values for the components of the extended Randles cell determined from the fit parameters for implanted, flexible, polyimide based probes.

\begin{tabular}{cccccc} 
& $\mathrm{R}_{\mathrm{s}}[\mathrm{k} \Omega]$ & $\mathrm{R}_{\mathrm{ct}}[\mathrm{k} \Omega]$ & $\mathrm{C}_{\mathrm{dl}}[\mathrm{nF}]$ & $\mathrm{R}_{\mathrm{m}}[\mathrm{k} \Omega]$ & $\mathrm{C}_{\mathrm{m}}[\mathrm{nF}]$ \\
\hline recording & $7.4 \pm 0.2$ & $17.9 \pm 0.9$ & $1.9 \pm 0.1$ & $342.7 \pm 14.3$ & $5.5 \pm 0.1$
\end{tabular}

stimulation $\quad 4.3 \pm 0.1 \quad 9.3 \pm 0.4 \quad 2.8 \pm 0.1 \quad 268.0 \pm 13.5 \quad 9.6 \pm 0.2$

The data obtained from the probes which were implanted in cerebral tissue for 6 month are shown as BODE and NYQUIST plot with mean value \pm standard deviation (Image 3 c). Here, the extended RANDLES cell circuit (Image 1) was used as a model to fit the impedance spectra and a goodness of fit of $26.84 \times 10^{-3}$ was obtained for recording- and $23.36 \times 10^{-3}$ for stimulation sites. The component values of the extended RANDLES cell circuit were determined from the fit parameters (Table 2).

Table 3 Area and $1 \mathrm{kHz}$ impedances for the microprobes used for impedance measurement in vitro and in vivo.

\begin{tabular}{cccc} 
probe & type & $\mathrm{A}$ & $\mathrm{Z}[\mathrm{k} \Omega]$ \\
\hline $\begin{array}{c}\text { metal based probes } \\
\text { in saline (Fig 2 A) }\end{array}$ & recording & 3600 & $99.4 \pm 20.3$ \\
& stimulation & 11300 & $62.7 \pm 11.1$ \\
$\begin{array}{c}\text { polyimide based probes } \\
\text { in saline (Fig 2 B) }\end{array}$ & recording & 400 & $306.2 \pm 51.7$ \\
& stimulation & 3850 & $125.4 \pm 17.1$ \\
$\begin{array}{c}\text { polyimide based probes } \\
\text { in saline (Fig 2 C) }\end{array}$ & recording & 225 & $388.3 \pm 74.1$ \\
$\begin{array}{c}\text { polyimide based probes } \\
\text { in tissue (Fig 2 C) }\end{array}$ & recording & 225 & $50.1 \pm 8.7$ \\
& stimulation & 2500 & $40.3 \pm 16.2$
\end{tabular}

The characteristic $1 \mathrm{kHz}$ impedance for all the different probe contact sites measured in saline electrolyte and tissue was obtained (Table 3 ). The $1 \mathrm{kHz}$ for all probe sites measured in saline electrolyte was plotted against the geometric surface area in a double logarithmic coordinate system. The data were fitted using a log-log fit (Image 4). 


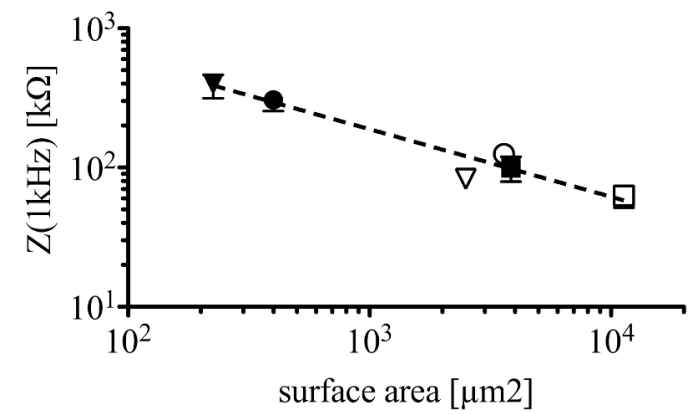

Image 4 Relationship between surface area of recording (filled) and stimulation (open) sites and $1 \mathrm{kHz}$ impedance at rigid (square) and two types of flexible probes (circle, triangle) in saline electrolyte. The dotted line shows the $\log -\log$ fit over all data points.

\section{Conclusion}

In the current study the frequency response of two different types of flexible, polyimide based probes was compared with rigid, metal based probes in saline electrolyte. The RANDLES cell equivalent circuit was used to describe the electrode-electrolyte interface and fitted well for metal based probes and for flexible probes at frequencies lower than $10 \mathrm{kHz}$. Comparing the $1 \mathrm{kHz}$ impedance of the different probe types in electrolyte leads to the conclusion that, at $1 \mathrm{kHz}$, the surface area of the contact site is the major determinant of the impedance and not so much the manufacturing process.

Further, flexible polyimide based probes were implanted into the cerebral tissue for 6 month and impedance spectra were recorded. Surprisingly, the $1 \mathrm{kHz}$ impedance of implanted probes was considerably lower as measured in saline electrolyte and the altered arrangement of reference and ground electrodes in vivo might play a huge role when impedance values are compared between in vivo and in vitro scenarios.

An extended RANDLES cell circuit was used to describe the electrode-tissue interface. A good fit for the frequency response of flexible probes after long term tissue implantation was obtained. We observed moderate $1 \mathrm{kHz}$ impedance of $47.5 \pm 11.4 \mathrm{k} \Omega$ after long term tissue implantation and conclude that the excessive increase of impedance after long term tissue implantation is avoidable when polyimide based probes are used.

\section{$5 \quad$ References}

[1] Patil, P. G., Turner, D. The development of brainmachine interface neuroprosthetic devices. Neurother, 2008, 5, 137-146

[2] Bedard, C., Kröger, H., Destexhe, A. Modeling extracellular field potentials and the frequency-filtering properties of extracellular space. Biophys J, 2004, 84, 1829 1842

[3] Berens, P., Logothetis, N. K., and Tolias, A. S. Local field potentials, BOLD and spiking activity - relationships and physiological mechanisms, Nature Prec, 2010, available from: http://hdl.handle.net/ 10101/npre.2010.5216.1

[4] McAdams, E. T., Lackermeier, A., McLaughlin, J. A., Macken, D., Jossinet, J. The linear and non-linear electrical properties of the electrode-electrolyte interface, Biosensors \& Bioelectronics, 1995, 10, 67-74

[5] Geddes, L. Historical evolution of circuit models for the electrode-electrolyte interface. Ann Biomed Eng, 1997, 25, 1-14

[6] Loveday, D., Peterson, P., Rodgers, B. Evaluation of Organic Coatings with Electrochemical Impedance Spectroscopy. JCT Coatings Tech, 2004, 46-52

[7] Wei, X. F., Grill, W. M. Impedance characteristics of deep brain stimulation electrodes in vitro and in vivo. $\mathrm{J}$ Neur Eng, 2009, 6(4), 9pp

[8] Yousif, N., Liu, X. Investigating the depth electrodebrain interface in deep brain stimulation using finite element models with graded complexity in structure and solution.J Neurosci Meth, 2009, 184(1), 142-151

[9] Polikov, V. S., Tresco, P., Reichert, W. M. Response of brain tissue to chronically implanted neural electrodes. J Neurosci Meth, 2005, 148, 1-18 